\title{
Modified endometriosis fertility index Is more accurate to predict the non-ART pregnancy rate following surgery: a cohort of Chinese women
}

\author{
Jiaying Fan ${ }^{1}$, Kang Qin ${ }^{1}$, Kuanrong $\mathrm{Li}^{2}$, Xiaojun $\mathrm{Li}^{2}$, Qingsheng Huang ${ }^{1}$, Yunsheng Liao ${ }^{1}$, \\ Huiying Liang ${ }^{1}$, Jingying Xie ${ }^{1}$, Yan Yang ${ }^{1}$, and Qingfeng $\mathrm{Li}^{2}$ \\ ${ }^{1}$ Affiliation not available \\ ${ }^{2}$ Guangzhou Women and Children's Medical Center, Guangzhou Medical University
}

April 28, 2020

\begin{abstract}
Objective: To examine whether a modified endometriosis fertility index (EFI) can better predict the rate of pregnancy without assisted reproductive technologies (ART) after laparoscopic surgery in infertile women with endometriosis. Design: Retrospective cohort study. Setting: A university medical center. Population: 564 infertile patients who received laparoscopic surgery for endometriosis. Methods: 472 patients were used to modify the EFI based on new, optimal cutoffs for its predictor variables. The predictive accuracy of the modified EFI was examined in the other 92 patients. Main outcome measures: post-surgical non-ART pregnancy. Results: Among the patients for the EFI modification, the multivariable Cox regression results showed that historical factors were more contribution in predicting non-ART pregnancy rate than surgical factors both in modified EFI (C-index: historical factors 0.617 vs surgical factors 0.558 ) and the original EFI (C-index: historical factors 0.600 vs surgical factors 0.549 ). No significant relationship between the prior pregnancy and post-operative non-ART pregnancy rates was detected by both modified EFI and original EFI ( $\mathrm{P}=0.530$ and 0.802 , respectively). In the patients for assessing the modified EFI, the predictive accuracy of two modified EFI models (C-index: 0.627 and 0.632, respectively) for non-ART pregnancy rates were superior to that of the original EFI (C-index: 0.602). Conclusions: A modified EFI based on population-specific optimal cutoffs and weight might be more suitable for estimating the rate of non-ART pregnancy after laparoscopic surgery in infertile women with endometriosis. Funding: This study received no external funding. Key words: endometriosis fertility index / pregnancy rate / predicting factors / model
\end{abstract}

\section{Introduction}

Endometriosis, a disease of unknown origin, affects $10 \%$ of women of reproductive age and about $50 \%$ of infertile women by damaging their pelvic cavity, ovaries and uterus. ${ }^{1,2}$ Pharmacotherapy is not effective. ${ }^{3,4}$ Surgery and assisted reproductive technologies (ART) can improve the treatment of endometriosis-accociated infertility. ${ }^{5-10}$ A recent study has reported possible increased cancer risk in children born to women utilizing ART in Denmark. ${ }^{11}$ Therefore, it is necessary that a tool should be available to predict the non-ART pregnancy rate after laparoacopic surgery in clinical practice.

The revised American Fertility Society score (r-AFS $)^{12}$ has not been shown to effectively predict postoperative pregnancy rate. ${ }^{13-16}$ Through adding clinical variables (age, duration of infertility, prior pregnancy) and surgical findings (least function score, LF), Adamson and Pasta developed the endometriosis fertility index (EFI) to predict non-ART pregnancy rate after surgery. ${ }^{17}$ Having been tested in the USA and validated in an external population, EFI has shown a moderate predictive accuracy. ${ }^{18-21}$ However, the contribution of each variable in EFI varies significantly in predicting the non-ART pregnancy rate after surgery in different populations. LF score, which accounts for $30 \%$ of EFI score, is considered the most decisive variable of 
pregnancy rate. ${ }^{17-19,21,}{ }^{22}$ However, the predictive efficiency of LF score did not appear as robust in two Asian cohorts. ${ }^{23},{ }^{24}$ Similarly, other variables in EFI also failed to predict the non-ART pregnancy rate after surgery in certain conditions. ${ }^{19,21}, 23-25$ Therefore, it is necessary to modify the original EFI to make it suitable to the Asian.

In the present study, we examined whether each variables of the EFI are suitable to predict the postoperative non-ART pregnancy rate in a Chinese cohort of infertile women with endometriosis. We identified the optimal cut-offs of EFI variables using the X-tile method ${ }^{26}$ and modified the EFI system through changing the groupings and the weights based on the optimal cutoffs and the contribution of six variables, respectively.

\section{Materials and Methods}

\section{Study population}

We identified 564 infertile patients (472 from 2014 to 2017 and 92 patients from 2018, respectively) with endometriosis who had undergone laparoscopic surgery as part of their work-up for infertility in the Department of Gynecology of the Guangzhou Women and Children's Medical Center. The diagnosis of peritoneal endometriosis was established according to histopathological characteristics or laparoscopic visualization of typical lesions. The diagnosis of ovarian endometrioma or deeply invasive endometriosis (DIE) was based on histopathology. DIE lesions were classified as deep invasive endometriotic lesions ([?] 5mm deep) or lesions involving bowel, bladder, ureter or other sites. ${ }^{27}$ Patients were excluded when they had other causes of infertility including ovulation disorder, bilateral tubal obstruction, congenital defects, intrauterine adhesion, endometrial hyperplasia, abnormal sexual function, adenomyosis, submucous myoma, Intramural myoma ([?] $3 \mathrm{~cm}$ ), underwent ART post-operatively immediately and abnormal semen of partners according to the WHO criteria. ${ }^{28}$ Patients were censored when lost to follow-up, had a subsequent surgery for recurrent endometriosis, had no plan for pregnancy or received ART, whichever came first.

\section{Treatment of infertility}

All patients underwent laparoscopic surgery and hysteroscopy simultaneously. Tubal patency was checked with methylene blue during the operation. During the laparoscopic surgery, all visible peritoneal lesions were excised or electrocoagulated. Endometrioma was treated by cystectomy using a careful stripping technique to minimize the decrease of the ovarian reserve. Deep invasive endometriosis (DIE) in the cul-de-sac and uterosacral ligament was removed thoroughly and bowel DIE was removed using shaved resection. Complete adhesionlysis was performed to restore the anatomic structure of pelvic organ and the cul-de-sac, and mobilize the tubes, fimbria and ovaries.

Post-operative treatments were based on the clinical situation of the patients. Pregnancy was defined by a $\mathrm{HCG}_{i} 25 \mathrm{IU} / \mathrm{L}$ or ultrasound visualization of one or more gestational sacs or definitive clinical signs of pregnancy. ${ }^{29}$ For patients who received post-operative Gonadotrophin-releasing hormone agonists (GnRHa), time to post-operative pregnancy was calculated as the interval between the date of the last injection and the date of the last menstrual period. For those who did not, it was calculated as the interval between the date of surgery and the date of the last menstrual period.

\section{Data collection}

Eligible patients were identified using the electronic medical record (EMR) and then contacted via telephone interview for further assessment of eligibility and data collection. The demographic data, reproductive history, endometriosis type and staging, EFI score (as described by Adamson and Pasta) ${ }^{17}$ and its variables were derived from the electronic medical record (EMR). Subsequent fertility information and pregnancy outcomes of the participants were derived from the EMR. If not, those were ascertained via telephone interview. When patients could not be reached after two telephone calls, text messages were sent. Women were asked to provide information regarding their pregnancy attempts, pregnancy outcomes and infertility treatment with or without ART. 


\section{Formulation and validation of Modified EFI}

To improve the predictive accuracy of EFI in Chinese patients, the EFI was modified by revising the cutoffs and weights of its six variables, based on the data of 434 patients recruited from 2014 to 2017 . Then, the efficiency of modified EFI was validated externally and compared with that of the original EFI in 85 patients recruited from 2018.

The five continuous variables (age, duration of infertility, least function score, r-AFS lesion score, and r-AFS total score) were categorized into three groups and their cut-offs were defined according to the maximum differences associated with post-operative pregnancy rates. Then, based on the new cut-offs of each variable, the three Kaplan-Merier curves of cumulative non-ART pregnancy rates were plotted. Similar curves were merged, and the variables was were dichotomized. We also tried to categorize prior pregnancy into three groups (prior live birth, prior pregnancy but no live birth, and no prior pregnancy). Finally, modified EFI was established on these variables of the original EFI with varying cut-offs and weights.

\section{Statistical analysis}

The X-tile method was used to select cut-offs of continuous variables associated with the maximum differences in non-ART pregnancy rates. ${ }^{26}$ The cumulative pregnancy rates were evaluated using Kaplan-Merier estimators and the Log-rank tests were used to compare the cumulative pregnancy rates among different subsets. Cox regression models were built to examine the EFI and its factors' predictive accuracy for post-operative non-ART pregnancy.

All statistical analyses were conducted with R software, version 3.6.1 (R Foundation for Statistical Computing, Vienna, Austria). A two-side $\mathrm{P}$ value of less than 0.05 was considered statistically significant.

\section{Results}

\section{Descriptive results and baseline characteristics}

Of the 472 eligible patients, from 2014 to 2017, who attempted non-ART pregnancy after laparoscopic surgical treatment of endometriosis, $434(91.9 \%)$ were contacted and agreed to participate in the study (Figure.1). The time between surgery and the telephone interview in the follow-up ranged from 18.4 to 67.4 months (median 37.7 months), and 255 (58.8\%) patients became pregnant without ART after surgery. Baseline characteristics of the patients in this study were summarized in Table 1 and the distribution of the original EFI, in 2014 to 2017 and in 2018, was shown in Figure S2 and Figure S3, respectively.

\section{Performance of original EFI and Its six variables}

The performance of six variables of original EFI in predicting non-ART pregnancy rate was showed in Table 2. According to the non-ART pregnancy rate Kaplan-Merier curves, prior pregnancy and the cutoffs of the least function score and AFS endometriosis score did not appear applicable in our study population, as evidenced by the crossed or overlapped curves (Figure. 2). In addition, patients aged 36-39 years showed lower pregnancy rate than those aged [?] 40 years (Figure. 2).

\section{New cutoffs and weights of variables in modified EFI}

Five continuous variables were categorized into three groups using X-tile to select cut-offs associated with the maximum difference in post-operative non-ART pregnancy rates. We found that the Kaplan-Merier curves of the three variables (least function score, AFS endometriosis score, and AFS total score) were overlapped (Figure S1). Therefore, these three variables were dichotomized (Figure. 2). When prior pregnancy was divided into three groups (non-pregnancy, pregnancy but no live birth and live birth), we observed the three curves was almost overlapped (Figure. 2) and no significant relationship between the prior pregnancy and post-operative non-ART pregnancy rates $(\mathrm{P}=0.802)$ (table 2$)$. The new cutoffs of variables in the modified EFI were listed in table 3.

The multivariable Cox regression results showed that historical factors were more predictive of non-ART pregnancy rate than surgical factors both in the original EFI (C-index: historical factors 0.600 vs surgical 
factors 0.549) and modified EFI (C-index: historical factors 0.617 vs surgical factors 0.558 ) (Table 2). The revised weights of the variables in the modified EFI were listed in table 3.

After re-grouping and re-weighting the EFI, two new EFI models were established (Table 3). The distributions of original EFI and modified EFI in patients recruited between 2014 and 2017 were showed in Figure $\mathrm{S} 2$.

\section{Efficiency comparison between modified EFI and original EFI}

As shown in Figure 1, the modified cutoffs of variables (prior pregnancy excluded) in EFI were more accurate to predict the cumulative non-ART pregnancy rates. The Cox proportional hazards models yielded the similar results, in which C-indexes of the re-categorized variables were all higher than those of the original variables (Table 2).

\section{External validation of two modified EFI models}

External validation in patients undergoing surgery during 2018 showed that the predictive accuracy of two modified EFI (C-index: 0.612 and 0.638, respectively) for non-ART pregnancy rates were superior to that of the original EFI (C-index: 0.560).

\section{Discussion}

In our study, we found the modified EFI is superior to the original EFI for predicting in the probability of non-ART pregnancy rate after laparoscopic surgical staging in patients endometriosis-associated infertility in China. In addition, we observed historical factors were more influential than surgical factors in determining the predictive value of EFI, and we found no significant relationship between "prior pregnancy", a variable in original EFI, and post-operative non-ART conception. To the best of our knowledge, this is the first attempt to modify the EFI to predict post-operative non-ART pregnancy rate in infertile patients with endometriosis.

In the EFI system developed by Adamson and Pasta ${ }^{17}$, the LF score makes the biggest contribution (about $30 \%$ ) in EFI score. ${ }^{18,19,21}$ However, this was not the case in our data and another two studies in Asian populations. ${ }^{23,24}$ The accuracy of EFI score is also adversely affected by the subjectivity of different surgeons and the interactions between its variables. For example, LF score is greatly affected by r-AFS lesion score and total $\mathrm{r}$-AFS score. In addition, age also influences LF score because the severity of endometriosis increases with age, which may reduce the effectiveness of surgery. ${ }^{30}$ Therefore, the contribution of LF score in the predictive value might be overestimated. On the other hand, there are few differences between LF 4 and LF 8. When LF is 4, 3 pathological conditions may occur. Among them, the patient with normal ovary and oviduct on one side, though those of the other side are dysfunctional, can still achieve pregnancy like those with LF score of $8 .{ }^{31}$ Even in the worst case, in which bilateral ovarian functions are moderate damaged by endometrioma because ovarian endometriosis is more common than oviduct endometriosis. In addition, laparoscopic cystectomy reduces oocyte quantity but not oocyte quality, which may also bring a chance of pregnancy. ${ }^{32,} 33$

The r-AFS classification system, based on endometriosis lesion, adhesion and cul-de-sac scores, is frequently used to appraise the severity of endometriosis and predict the potential recurrence after surgery. However, it is not useful in predicting post-operative pregnancy ${ }^{13-16}$. Endometriosis mainly invades the ovaries and peritoneum. Patients with peritoneum endometriosis (maximal r-AFS 6), unilateral endometrioma (maximal r-AFS 20) and both (maximal r-AFS 26) may still show a normal rate of post-operative pregnancy, due to the normal ovarian function in at least one side. ${ }^{31}$ But in those with bilateral deep endometrioma $(1 \mathrm{~cm}$ in diameter or multilocular cysts, maximal r-AFS [?] 32), the post-operative pregnancy rate drops obviously. ${ }^{34}$ Our data showed the optimal cut-off of the r-AFS lesion should be 32 to meet the clinical requirements. ${ }^{3,35 \text {, }}$ 36

Age is an independent risk factor for endometriosis and infertility. Women aged $>30$ years experience increased probability of infertility. ${ }^{37}$ The age-related infertility may be caused by the decrease in the number of primordial follicles ${ }^{38}$ as well asoocyte quality and quantity. ${ }^{33}$ The risk of infertility following laparoscop- 
iclly confirmed endometriosis was significantly elevated among women aged $<35$ years. ${ }^{39}$ In those aged [?] 35 years, the quality and quantity of oocytes decrease rapidly, increasing the risk of other endocrine disorders, and infertility. ${ }^{38,40}$ Additionally, as age increases, endometrioma becomes more multilocular; ${ }^{41}$ in this condition, surgery-caused damage to ovarian tissue aggravates, further diminishing the ovarian reserve. ${ }^{42,43}$ Therefore, our data suggested that the age cut-offs in EFI should be changed to 30 and 35 .

Longer duration of infertility was associated with lower pregnancy rate after surgery. ${ }^{13}$ As the time of infertility extends, endometriosis worsens and pelvic anatomical structure degenerates, all increasing the difficulty of surgery and reducing post-operative the chance of pregnancy. Even in patients suffering from stages I and II endometriosis, the inflammatory mediators in the pelvic cavity increase with longer duration of infertility, consequently interfering with sperm-oocyte interaction ${ }^{1,10}$ and bringing with subtle lesions in the distal tube, such as fimbrial agglutination, fimbrial phimosis, fimbrial blunting, diverticulum, accessory ostium, and convoluted oviduct. ${ }^{44,} 45$ These pathological changes further decreased the pregnancy rate. In our study, duration of infertility is the most relative to the EFI score, which the same result were observed in Hobo et al report. ${ }^{23}$ Therefore, we gave three groups to this variable in the modified EFI.

Though high gravidity and parity can decrease the incidence of endometriosis, ${ }^{46}$ pregnancy is also affected by many other factors, like personal plan. Prescott et $\mathrm{al}^{39}$ prospectively found that primary infertility and secondary infertility bring an equal risk to pregnancy in women with endometriosis. However, another study indicated that prior reproductive history may be informative for predicting fecundity and pregnancy outcomes in women with following endometriosis. ${ }^{47}$ Our results showed pregnancy history and live birth were not relevant with the post-operative non-ART pregnancy rate, which in agreement with some external validations. ${ }^{21,23,48}$ This finding may be explained by the reproductive policies, like family planning.

This study had several limitations. First, the variables were modified from those in the original EFI, and their inter-dependence has not been eliminated. Second, our study was a retrospective design and the size of sample might be not enough big to establish the model. Anyway, our study included almost all the infertile women with endometriosis who underwent laparoscopic fertility-sparing surgery in our center from January 2014 to December 2018. Finally, our study, accomplished in a single center in southern China, was not representative enough.

\section{Conclusions}

Our modified EFI is a suitable prediction tool for the non-ART pregnancy rate following the laparoscopic surgery in infertile Chinese women with endometriosis. Our efforts may encourage the development of more precise tools for predicting non-ART pregnancy rate in these women.

\section{Acknowledgements}

We warmly thank Ms. Jiexin Zhang and Ms. Tong Wu for performing the post-operative telephone interview.

\section{Author contributions}

Jiaying Fan, Kang Qin, Qingfeng Li, Kuanrong Li, Qingsheng Huang and Huiying Liang designed the study. Xiaojun Li, Yunsheng Liao Jingying Xie and Yan Yang prepared the data. Kang Qin performed the statistical analysis. Jiaying Fan and Kang Qin drafted the manuscript. Qingfeng Li, Kuanrong Li and Abraham N Morse reviewed the manuscript and contributed important intellectual inputs. All the authors read and approved the final version of the manuscript.

\section{Declaration of interest}

All authors declare that they have no competing interests. Completed disclosure of interest forms are available to view online as supporting information.

\section{Details of ethics interest}

This study was approved by the Guangzhou Women and Children's Medical Center Ethics Committee (No. 2017102709) and written informed consent was obtained from all participants. 


\section{Funding}

This study received no external funding.

\section{References}

1. de Ziegler D, Borghese B, Chapron C. Endometriosis and infertility: pathophysiology and management. Lancet. 2010 Aug 28;376(9742):730-8.

2. Eisenberg VH, Weil C, Chodick G, Shalev V. Epidemiology of endometriosis: a large population-based database study from a healthcare provider with 2 million members. BJOG : an international journal of obstetrics and gynaecology. 2018 Jan;125(1):55-62.

3. Dunselman GA, Vermeulen N, Becker C, Calhaz-Jorge C, D'Hooghe T, De Bie B, et al. ESHRE guideline: management of women with endometriosis. Human reproduction. 2014 Mar;29(3):400-12.

4. Hughes E, Brown J, Collins JJ, Farquhar C, Fedorkow DM, Vandekerckhove P. Ovulation suppression for endometriosis. The Cochrane database of systematic reviews. 2007 Jul 18(3):CD000155.

5. Chern CJ, Beutler E. Biochemical and electrophoretic studies of erythrocyte pyridoxine kinase in white and black Americans. Am J Hum Genet. 1976 Jan;28(1):9-17.

6. Marcoux S, Maheux R, Berube S. Laparoscopic surgery in infertile women with minimal or mild endometriosis. Canadian Collaborative Group on Endometriosis. N Engl J Med. 1997 Jul 24;337(4):217-22.

7. Parazzini F. Ablation of lesions or no treatment in minimal-mild endometriosis in infertile women: a randomized trial. Gruppo Italiano per lo Studio dell'Endometriosi. Human reproduction. 1999 May;14(5):13324 .

8. Darai E, Marpeau O, Thomassin I, Dubernard G, Barranger E, Bazot M. Fertility after laparoscopic colorectal resection for endometriosis: preliminary results. Fertility and sterility. 2005 Oct;84(4):945-50.

9. Meuleman C, Tomassetti C, Wolthuis A, Van Cleynenbreugel B, Laenen A, Penninckx F, et al. Clinical outcome after radical excision of moderate-severe endometriosis with or without bowel resection and reanastomosis: a prospective cohort study. Ann Surg. 2014 Mar;259(3):522-31.

10. de Ziegler D, Pirtea P, Carbonnel M, Poulain M, Cicinelli E, Bulletti C, et al. Assisted reproduction in endometriosis. Best practice \& research Clinical endocrinology \& metabolism. 2019 Feb;33(1):47-59.

11. Hargreave M, Jensen A, Hansen MK, Dehlendorff C, Winther JF, Schmiegelow K, et al. Association Between Fertility Treatment and Cancer Risk in Children. Jama. 2019 Dec 10;322(22):2203-10.

12. Revised American Society for Reproductive Medicine classification of endometriosis: 1996. Fertility and sterility. 1997 May;67(5):817-21.

13. Adamson GD, Hurd SJ, Pasta DJ, Rodriguez BD. Laparoscopic endometriosis treatment: is it better? Fertility and sterility. 1993 Jan;59(1):35-44.

14. Guzick DS, Silliman NP, Adamson GD, Buttram VC, Jr., Canis M, Malinak LR, et al. Prediction of pregnancy in infertile women based on the American Society for Reproductive Medicine's revised classification of endometriosis. Fertility and sterility. 1997 May;67(5):822-9.

15. Vercellini P, Fedele L, Aimi G, De Giorgi O, Consonni D, Crosignani PG. Reproductive performance, pain recurrence and disease relapse after conservative surgical treatment for endometriosis: the predictive value of the current classification system. Human reproduction. 2006 Oct;21(10):2679-85.

16. Adamson GD. Endometriosis classification: an update. Current opinion in obstetrics \& gynecology. 2011 Aug;23(4):213-20.

17. Adamson GD, Pasta DJ. Endometriosis fertility index: the new, validated endometriosis staging system. Fertility and sterility. 2010 Oct;94(5):1609-15. 
18. Boujenah J, Bonneau C, Hugues JN, Sifer C, Poncelet C. External validation of the Endometriosis Fertility Index in a French population. Fertility and sterility. 2015 Jul;104(1):119-23 e1.

19. Tomassetti C, Geysenbergh B, Meuleman C, Timmerman D, Fieuws S, D'Hooghe T. External validation of the endometriosis fertility index (EFI) staging system for predicting non-ART pregnancy after endometriosis surgery. Human reproduction. 2013 May;28(5):1280-8.

20. Zhang X, Liu D, Huang W, Wang Q, Feng X, Tan J. Prediction of Endometriosis Fertility Index in patients with endometriosis-associated infertility after laparoscopic treatment. Reprod Biomed Online. 2018 Jul;37(1):53-9.

21. Li X, Zeng C, Zhou YF, Yang HX, Shang J, Zhu SN, et al. Endometriosis Fertility Index for Predicting Pregnancy after Endometriosis Surgery. Chinese medical journal. 2017 Aug 20;130(16):1932-7.

22. Maheux-Lacroix S, Nesbitt-Hawes E, Deans R, Won H, Budden A, Adamson D, et al. Endometriosis fertility index predicts live births following surgical resection of moderate and severe endometriosis. Human reproduction. 2017 Nov 1;32(11):2243-9.

23. Hobo R, Nakagawa K, Usui C, Sugiyama R, Ino N, Motoyama H, et al. The Endometriosis Fertility Index Is Useful for Predicting the Ability to Conceive without Assisted Reproductive Technology Treatment after Laparoscopic Surgery, Regardless of Endometriosis. Gynecologic and obstetric investigation. 2018;83(5):4938.

24. Kim JS, Lee CW, Yun J, Lee JH, Yun BH, Park JH, et al. Use of the Endometriosis Fertility Index to Predict Natural Pregnancy after Endometriosis Surgery: A Single-Center Study. Gynecologic and obstetric investigation. 2019;84(1):86-93.

25. Boujenah J, Cedrin-Durnerin I, Herbemont C, Bricou A, Sifer C, Poncelet C. Use of the endometriosis fertility index in daily practice: A prospective evaluation. European journal of obstetrics, gynecology, and reproductive biology. 2017 Dec;219:28-34.

26. Camp RL, Dolled-Filhart M, Rimm DL. X-tile: a new bio-informatics tool for biomarker assessment and outcome-based cut-point optimization. Clinical cancer research : an official journal of the American Association for Cancer Research. 2004 Nov 1;10(21):7252-9.

27. Johnson NP, Hummelshoj L, Adamson GD, Keckstein J, Taylor HS, Abrao MS, et al. World Endometriosis Society consensus on the classification of endometriosis. Human reproduction. 2017 Feb;32(2):315-24.

28. Ford WC. Comments on the release of the 5th edition of the WHO Laboratory Manual for the Examination and Processing of Human Semen. Asian J Androl. 2010 Jan;12(1):59-63.

29. Zegers-Hochschild F, Adamson GD, de Mouzon J, Ishihara O, Mansour R, Nygren K, et al. The International Committee for Monitoring Assisted Reproductive Technology (ICMART) and the World Health Organization (WHO) Revised Glossary on ART Terminology, 2009. Human reproduction. 2009 Nov;24(11):26837 .

30. Brosens I, Gordts S, Benagiano G. Endometriosis in adolescents is a hidden, progressive and severe disease that deserves attention, not just compassion. Human reproduction. 2013 Aug;28(8):2026-31.

31. Aboulghar MA, Mansour RT, Serour GI. Spontaneous intrauterine pregnancy following salpingectomy for a unilateral hydrosalpinx. Human reproduction. 2002 Apr;17(4):1099-100.

32. Ragni G, Somigliana E, Benedetti F, Paffoni A, Vegetti W, Restelli L, et al. Damage to ovarian reserve associated with laparoscopic excision of endometriomas: a quantitative rather than a qualitative injury. American journal of obstetrics and gynecology. 2005 Dec;193(6):1908-14.

33. de Ziegler D, Gayet V, Aubriot FX, Fauque P, Streuli I, Wolf JP, et al. Use of oral contraceptives in women with endometriosis before assisted reproduction treatment improves outcomes. Fertility and sterility. 2010 Dec;94(7):2796-9. 
34. Orazov MR, Radzinsky VY, Ivanov, II, Khamoshina MB, Shustova VB. Oocyte quality in women with infertility associated endometriosis. Gynecological endocrinology : the official journal of the International Society of Gynecological Endocrinology. 2019;35(sup1):24-6.

35. Jacobson TZ, Barlow DH, Koninckx PR, Olive D, Farquhar C. Laparoscopic surgery for subfertility associated with endometriosis. The Cochrane database of systematic reviews. 2002(4):CD001398.

36. Practice Committee of the American Society for Reproductive M. Endometriosis and infertility: a committee opinion. Fertility and sterility. 2012 Sep;98(3):591-8.

37. Steiner AZ, Jukic AM. Impact of female age and nulligravidity on fecundity in an older reproductive age cohort. Fertility and sterility. 2016 Jun;105(6):1584-8 e1.

38. Faddy MJ, Gosden RG, Gougeon A, Richardson SJ, Nelson JF. Accelerated disappearance of ovarian follicles in mid-life: implications for forecasting menopause. Human reproduction. 1992 Nov;7(10):1342-6.

39. Prescott J, Farland LV, Tobias DK, Gaskins AJ, Spiegelman D, Chavarro JE, et al. A prospective cohort study of endometriosis and subsequent risk of infertility. Human reproduction. 2016 Jul;31(7):1475-82.

40. American College of O, Gynecologists Committee on Gynecologic P, Practice C. Female age-related fertility decline. Committee Opinion No. 589. Fertility and sterility. 2014 Mar;101(3):633-4.

41. Guerriero S, Van Calster B, Somigliana E, Ajossa S, Froyman W, De Cock B, et al. Age-related differences in the sonographic characteristics of endometriomas. Human reproduction. 2016 Aug;31(8):1723-31.

42. Sanchez AM, Vanni VS, Bartiromo L, Papaleo E, Zilberberg E, Candiani M, et al. Is the oocyte quality affected by endometriosis? A review of the literature. Journal of ovarian research. 2017 Jul 12;10(1):43.

43. Muzii L, Di Tucci C, Di Feliciantonio M, Galati G, Di Donato V, Musella A, et al. Antimullerian hormone is reduced in the presence of ovarian endometriomas: a systematic review and meta-analysis. Fertility and sterility. 2018 Oct;110(5):932-40 e1.

44. Abuzeid MI, Mitwally MF, Ahmed AI, Formentini E, Ashraf M, Abuzeid OM, et al. The prevalence of fimbrial pathology in patients with early stages of endometriosis. Journal of minimally invasive gynecology. 2007 Jan-Feb;14(1):49-53.

45. Zheng X, Han H, Guan J. Clinical features of fallopian tube accessory ostium and outcomes after laparoscopic treatment. International journal of gynaecology and obstetrics: the official organ of the International Federation of Gynaecology and Obstetrics. 2015 Jun;129(3):260-3.

46. Peterson CM, Johnstone EB, Hammoud AO, Stanford JB, Varner MW, Kennedy A, et al. Risk factors associated with endometriosis: importance of study population for characterizing disease in the ENDO Study. American journal of obstetrics and gynecology. 2013 Jun;208(6):451 e1-11.

47. Buck Louis GM, Backonja U, Schliep KC, Sun L, Peterson CM, Chen Z. Women's Reproductive History Before the Diagnosis of Incident Endometriosis. Journal of women's health. 2016 Oct;25(10):1021-9.

48. Li X, Xu X, Rao X, Tian Y, Yi W. Chemical remodeling cell surface glycans for immunotargeting of tumor cells. Carbohydrate research. 2017 Nov 27;452:25-34.

\section{Figure le gends}

Figure 1. Flow chart of study population.

Figure 2. Kaplan-Meier estimators for the cumulative non-ART pregnancy rate as a function of the original (dashed lines) and modified (solid lines) EFI factors in patients between 2014 and 2017.

Supplemental Figure 1. Kaplan-Meier estimators for the cumulative non-ART pregnancy rate as a function of the three-categorized (A) least function score, (B) AFS endometriosis score, and (C) AFS Total score in patients between 2014 and 2017. 
Supplemental Figure 2. Distribution of original EFI and modified version A and B of EFI in patients between 2014 and 2017.

Supplemental Figure 3. Distribution of original EFI and modified version A and B of EFI in patients of 2018.

TABLE 1 Characteristics of study population.

\begin{tabular}{|c|c|c|c|}
\hline Variables, $\mathrm{n}(\%)$ & $\begin{array}{l}\text { Study population } \\
\text { During 2014-2017 }(\mathrm{n}= \\
434)\end{array}$ & $\begin{array}{l}\text { Study population } \\
\text { In } 2018(\mathrm{n}=85)\end{array}$ & $\mathrm{P}$ \\
\hline $\begin{array}{l}\mathrm{BMI}\left(\mathrm{kg} / \mathrm{m}^{2}\right) \\
\operatorname{mean} \pm \mathrm{sd}\end{array}$ & $21.07 \pm 2.91$ & $21.22 \pm 2.43)$ & 0.649 \\
\hline Age (years) & & & $0.615^{*}$ \\
\hline [?]35 & $350(80.6)$ & $65(76.5)$ & \\
\hline $36-39$ & $59(13.6)$ & $14(16.5)$ & \\
\hline$[?] 40$ & $25(5.8)$ & $6(7.1)$ & \\
\hline $\begin{array}{l}\text { Duration of infertility } \\
\text { (month) }\end{array}$ & & & $0.414^{*}$ \\
\hline [?]36 & $329(75.8)$ & $61(71.8)$ & \\
\hline$>36$ & $105(24.2)$ & $24(28.2)$ & \\
\hline Previous pregnancy & $208(47.9)$ & $39(45.9)$ & $0.812^{*}$ \\
\hline $\mathrm{LF}$ & & & $0.211^{*}$ \\
\hline $7-8$ & $58(13.4)$ & $11(12.9)$ & \\
\hline $4-6$ & $285(65.7)$ & $63(74.1)$ & \\
\hline $1-3$ & $91(21.0)$ & $11(12.9)$ & \\
\hline $\begin{array}{l}\text { r-ASRM endometriosis } \\
\text { score }\end{array}$ & & & $0.543^{*}$ \\
\hline$<16$ & $263(60.6)$ & $55(64.7)$ & \\
\hline$[?] 16$ & $171(39.4)$ & $30(35.3)$ & \\
\hline r-ASRM total score & & & $0.166^{*}$ \\
\hline$<71$ & $379(87.3)$ & $69(81.2)$ & \\
\hline$[?] 71$ & $55(12.7)$ & $16(18.8)$ & \\
\hline EFI & & & $0.368^{*}$ \\
\hline $0-4$ & $32(7.4)$ & $8(9.4)$ & \\
\hline $5-6$ & $100(23.0)$ & $26(30.6)$ & \\
\hline $7-8$ & $202(46.5)$ & $35(41.2)$ & \\
\hline $9-10$ & $100(23.0)$ & $16(18.8)$ & \\
\hline \multicolumn{4}{|l|}{ Pathology type } \\
\hline Peritoneal & $320(73.7)$ & $56(65.9)$ & $0.146^{*}$ \\
\hline Ovarian & $189(43.5)$ & $38(44.7)$ & $0.905^{*}$ \\
\hline DIE & $119(27.4)$ & $12(14.1)$ & $0.009^{*}$ \\
\hline r-ASRM & & & $0.243^{*}$ \\
\hline I & $189(43.5)$ & $37(43.5)$ & \\
\hline II & $38(8.8)$ & $10(11.8)$ & \\
\hline III & $104(24.0)$ & $13(15.3)$ & \\
\hline IV & $103(23.7)$ & $25(29.4)$ & \\
\hline
\end{tabular}

Abbreviations: EFI, Endometriosis Fertility Index; LF, least function score; DIE, deep infiltrating endometriosis; r-ASRM, revised American Society for Reproductive Medicine; ART, assisted reproductive technology. 
* $\mathrm{P}$ values were calculated using Fisher's exact test.

TABLE 2 Overview of the performance of original and modified EFI predicting non-ART pregnancy rate in patients between 2014 and 2017

\begin{tabular}{|c|c|c|c|c|c|}
\hline Factor in Cox model & $\chi^{2}$ & $\mathrm{df}$ & $\mathrm{P}$ & $\mathrm{R}^{2}$ & C-index $(95 \% \mathrm{CI})$ \\
\hline EFI continuous (linear, original) & 39.50 & 1 & 0.000 & 0.087 & $0.605(0.570-0.640)$ \\
\hline EFI continuous (linear, modified)a & 58.33 & 1 & $<0.001$ & 0.126 & $0.627(0.592-0.662)$ \\
\hline EFI continuous (linear, modified) ${ }^{\mathrm{b}}$ & 60.24 & 1 & $<0.001$ & 0.130 & $0.632(0.597-0.667)$ \\
\hline All historical factors (original) & 42.30 & 4 & 0.000 & 0.093 & $0.600(0.565-0.635)$ \\
\hline Age (original, [?]35; 36-39; [?]40) & 19.38 & 2 & 0.000 & 0.044 & $0.549(0.524-0.574)$ \\
\hline Years infertile (original, $[?] 3 ;>3$ ) & 25.01 & 1 & 0.000 & 0.056 & $0.566(0.541-0.591)$ \\
\hline ncy (original, yes; no) & 0.40 & 1 & 0.530 & 0.001 & $0.509(0.476-0.542)$ \\
\hline factors (modified) & 47.58 & 5 & $<0.001$ & 0.104 & $0.617(0.582-0.652)$ \\
\hline ed, [?]30; 31-35; [?]36) & 25.54 & 2 & $<0.001$ & 0.057 & $0.576(0.543-0.609)$ \\
\hline Years infertile (modified, [?]2; 2-3; [?]3) & 27.70 & 2 & $<0.001$ & 0.062 & $0.587(0.554-0.620)$ \\
\hline ified, live birth; yes but 1 & 0.44 & 2 & 0.802 & 0.001 & $0.545)$ \\
\hline 1) & 15.20 & 4 & 0.004 & 0.034 & $-0.586)$ \\
\hline Leas & 8.40 & 2 & 0 & 0.019 & $-0.563)$ \\
\hline nal; < 16; [?] 16) & 3.60 & 1 & 0 . & 08 & $3-0.565)$ \\
\hline tal score (original, < 71; [?] 71) & 12.56 & 1 & 0.0 & 0.029 & $2-0.556)$ \\
\hline All surgical factors (modified) & 20.63 & 3 & & 0.046 & $31-0.585)$ \\
\hline Least func & 7.93 & 1 & 0.005 & 0.018 & $0.525(0.503-0.547)$ \\
\hline modified, < $32 ;$ [?] 32) & 12.69 & 1 & $<0.001$ & 0.029 & $0.538(0.518-0.558)$ \\
\hline AFS Total score (modified, $<65 ;$ [?] 65) & 14.17 & 1 & $<0.001$ & 0.032 & $0.541(0.519-0.563)$ \\
\hline
\end{tabular}

a year of infertile was re-categorized into three groups and assigned 3, 2, and 0, respectively. Meanwhile, least function score was dichotomized and assigned 2 and 0 , respectively. See version A of modified EFI in table 3 for details.

b Prior pregnancy was excluded from the EFI score and the age was re-categorized into three groups and assigned 3,2, and 0, respectively. Others were same to the revised version A of EFI. See version B of modified EFI in table 3 for details.

Table 3 Two modified versions of the EFI calculator

\begin{tabular}{|c|c|c|c|}
\hline Modified EFI & Modified EFI Modified EFI & Modified EFI & Modified EFI \\
\hline Version A & Version A & Version B & Version B \\
\hline Factor & Points & Factor & Points \\
\hline Age (year) & & Age (year) & $? \gtreqless ?$ \\
\hline 30 & 2 & [?]30 & 3 \\
\hline $31-35$ & 1 & $31-35$ & $2 ? \gtreqless ?$ \\
\hline 36 & 0 & [?]36 & 0 \\
\hline Years infertile & & Years infertile & \\
\hline$<2$ & 3 & $<2$ & 3 \\
\hline $2-3$ & 2 & $2-3$ & 2 \\
\hline$>3$ & 0 & $>3$ & 0 \\
\hline Prior pregnancy & & Least function score & \\
\hline Yes & 1 & $<4$ & 2 \\
\hline No & 0 & {$[?] 4$} & 0 \\
\hline Least function score & & AFS Endometriosis score & \\
\hline$<4$ & 2 & $<32$ & $1 ? \gtreqless ?$ \\
\hline
\end{tabular}


4

AFS Endometriosis score

$<32$

32

AFS Total score

$<65$

65
[?]32

AFS Total score

$<65$

[?]65

$1 ? \dot{\imath} ?$

0

$? \dot{i} ?$

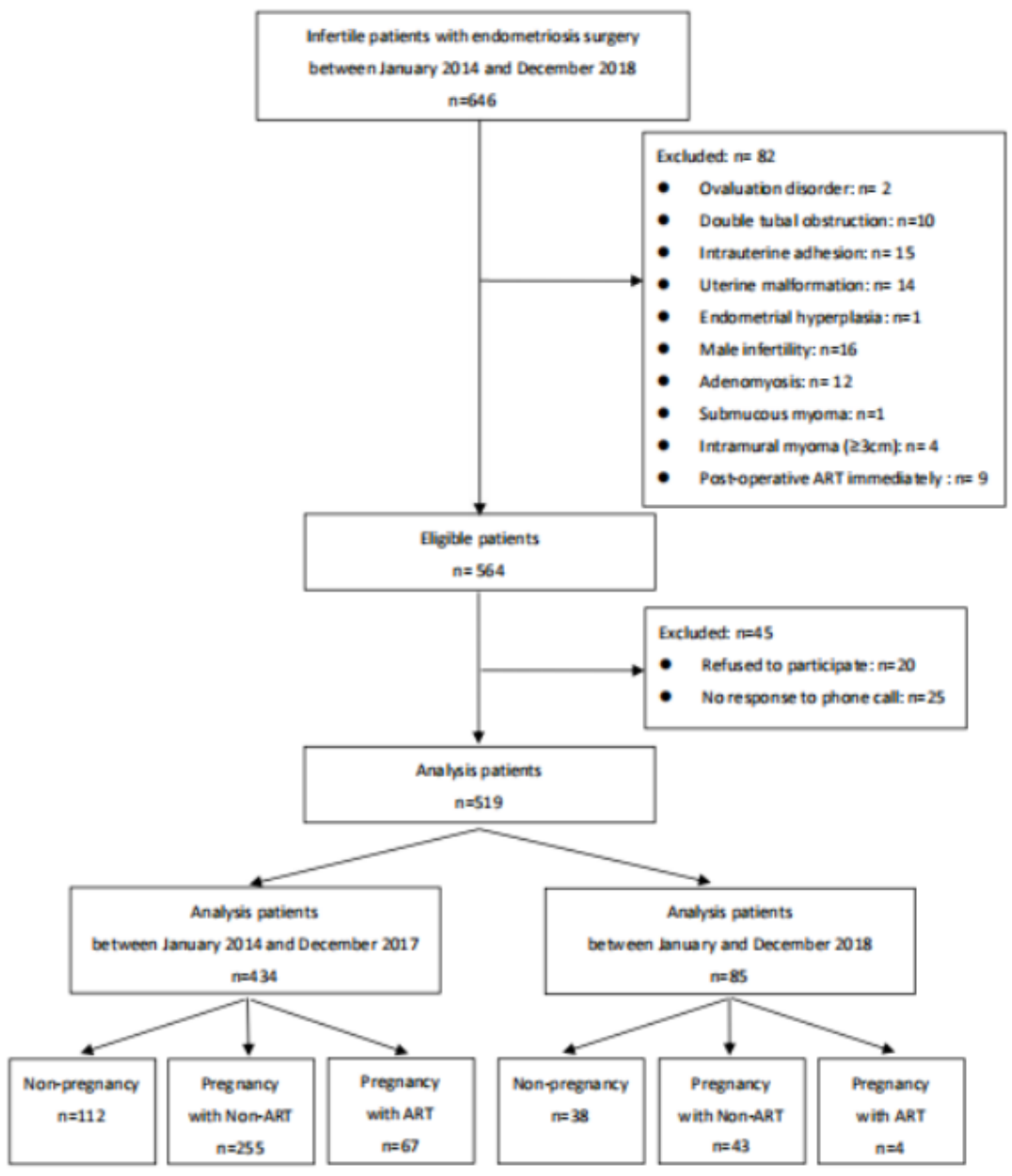


(A)

- - Age $\leq 35$ years - - Age $=36-39$ years - - Age $\geq 40$ years - Age $\leq 30$ years - Age $=31-35$ years - Age $\geq 36$ years

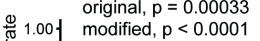

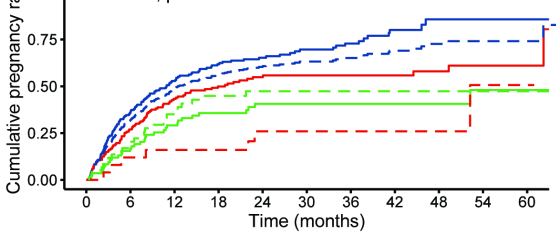

(C) - - Prior pregnancy = Yes - - Prior pregnancy $=$ No - Prior live birth - Prior pregnancy but no live birth - No prior pregnancy original, $p=0.53$ đٓ 1.00 modified, $p=0.80$

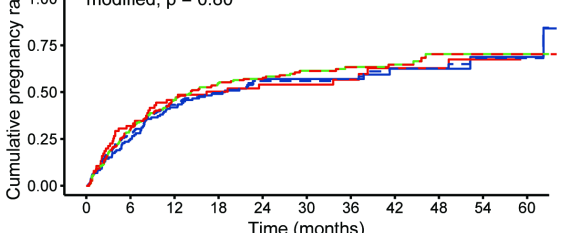

(E)

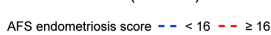

original, $p=0.06$

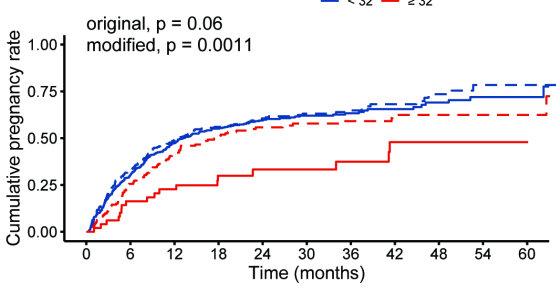

(B) - - Years infertile $\leq 3 \quad--$ Years infertile $>3$

- Years infertile $<2-$ Years infertile $=2-3-$ Years infertile $>3$ original, $p<0.0001$

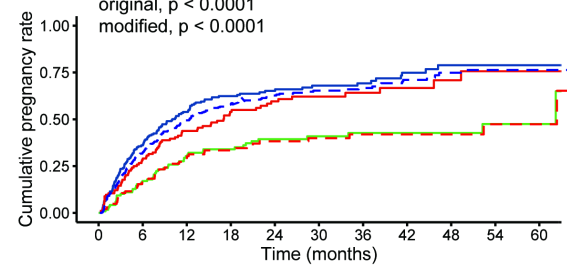

(D)

Least function score - - 1-3 - - 4-6 - - 7-8

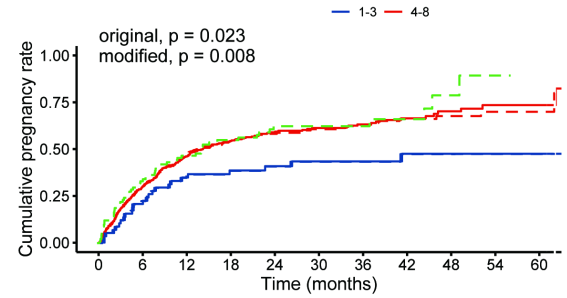

(F)

AFS total score - - < $<11--\geq 7$

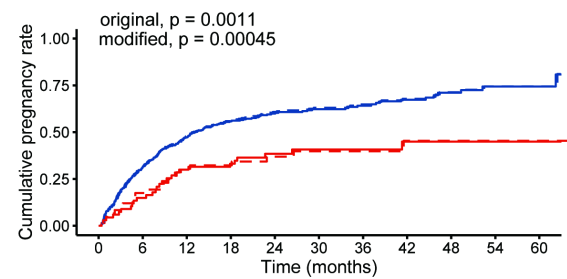


(A) Least function score $-0-3-4-5-8$

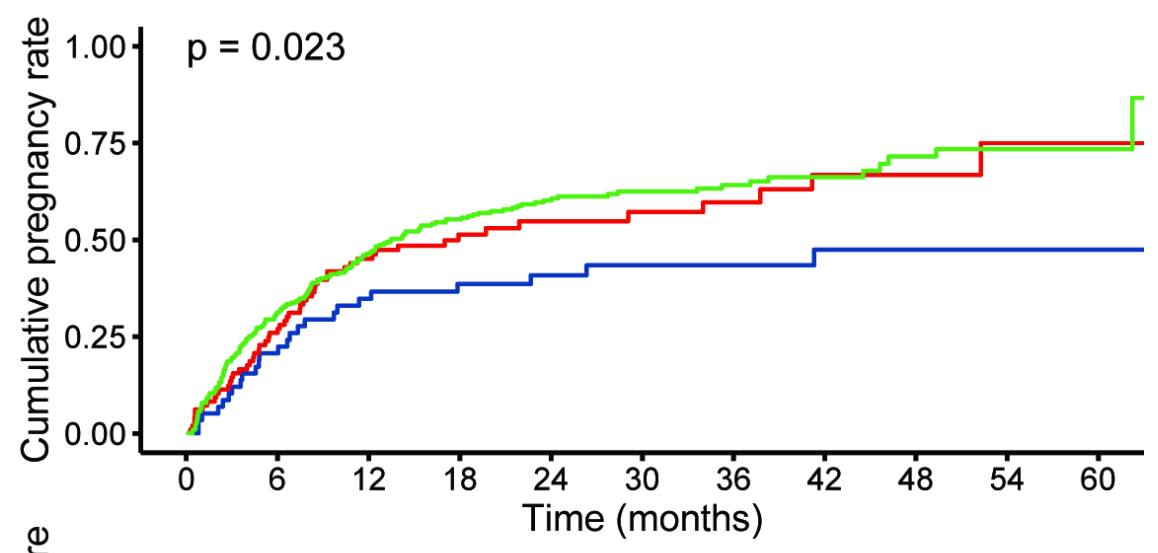

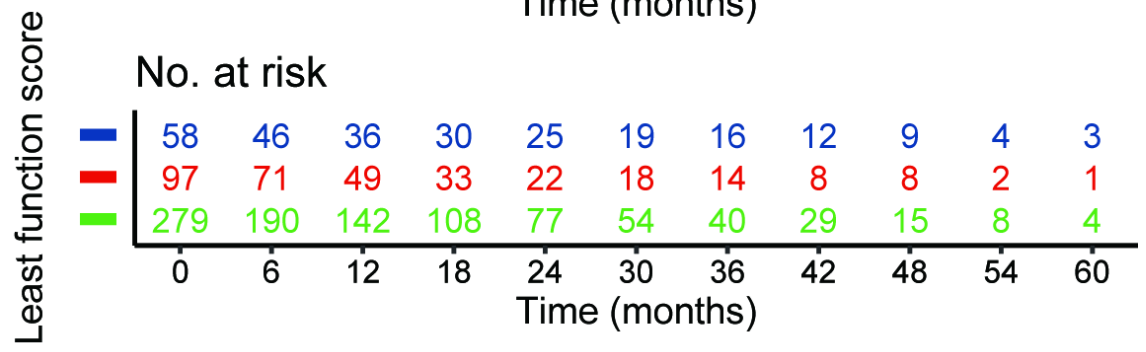

(B) AFS endometriosis score $-<16-16-31-\geq 32$
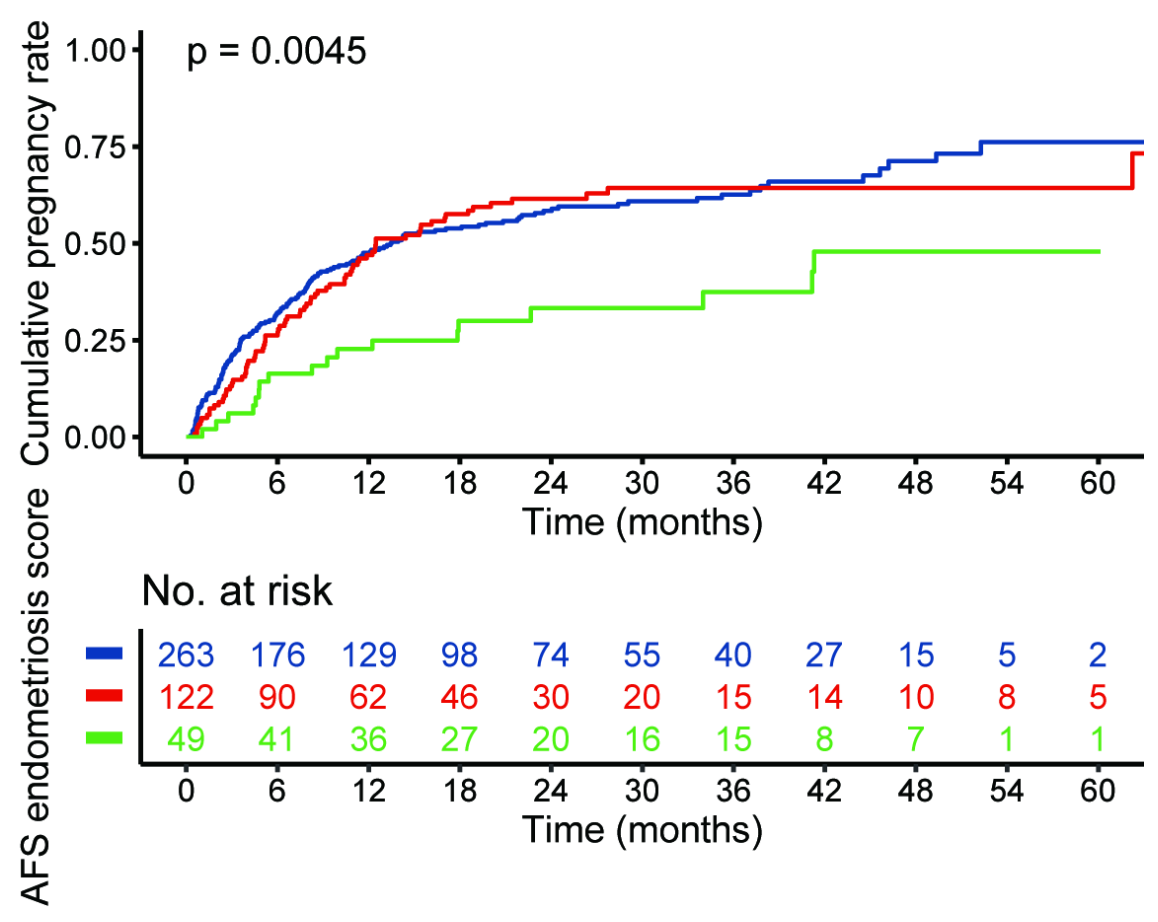

(C)

AFS total score $-<18-18-64-\geq 65$

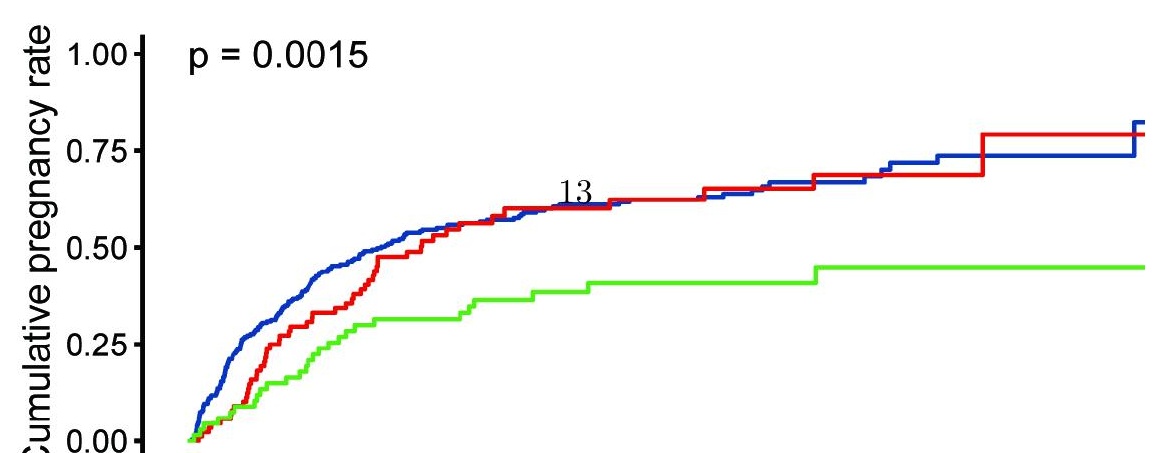




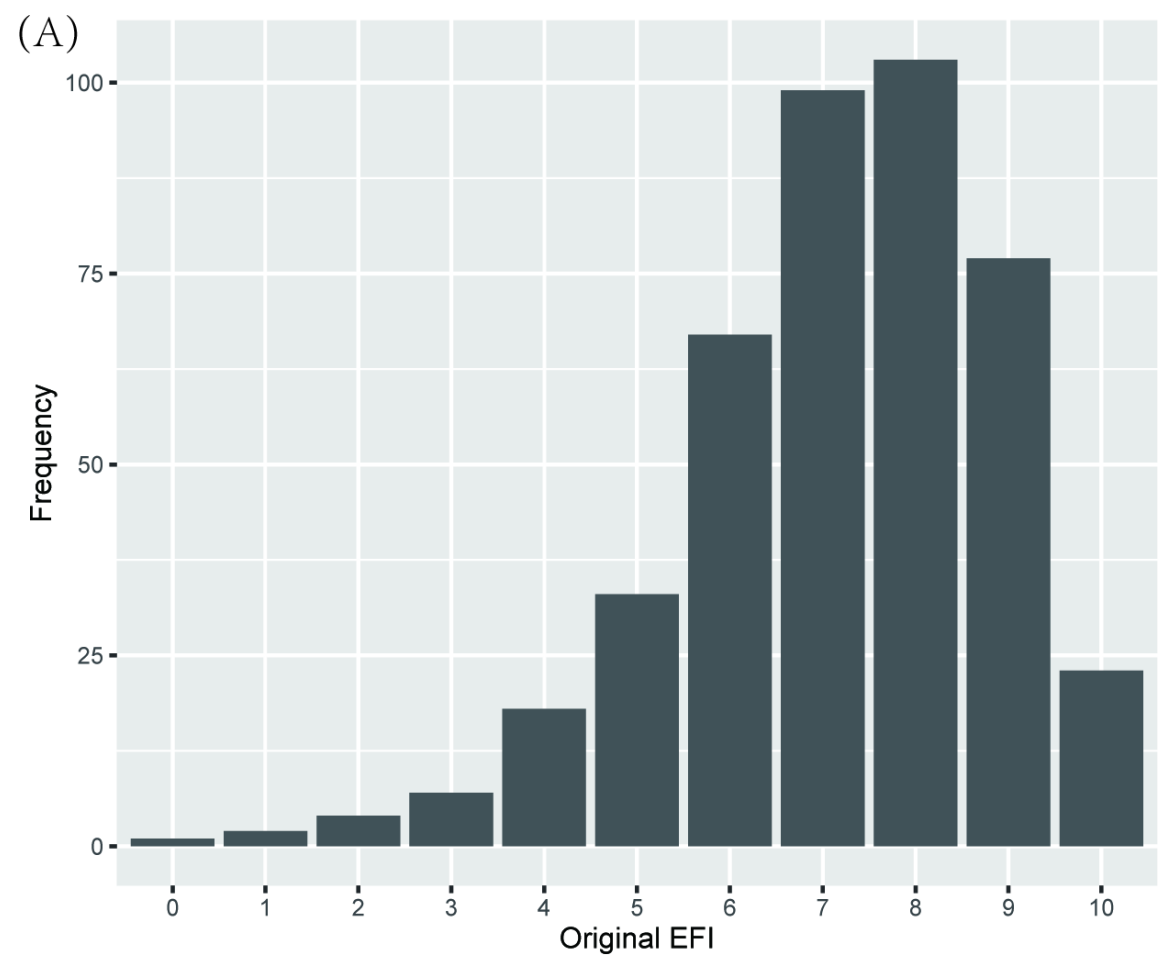

(B)

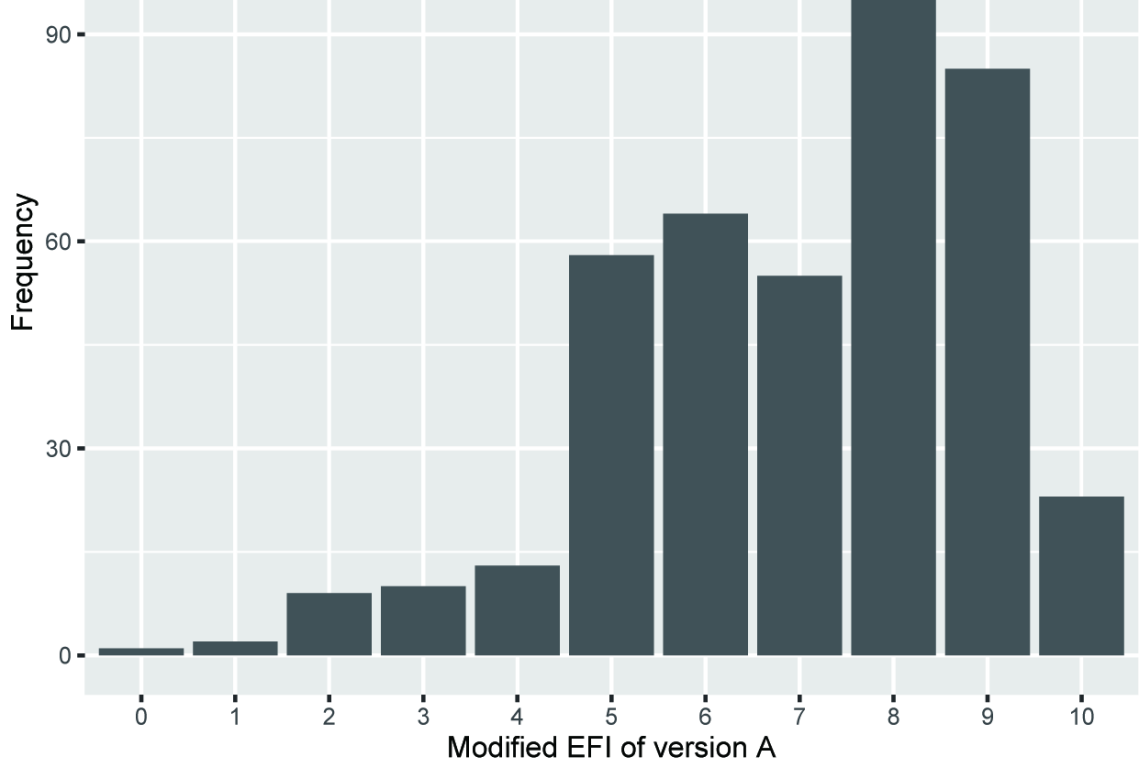

(C) 100 -

75 15

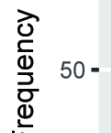


(A)

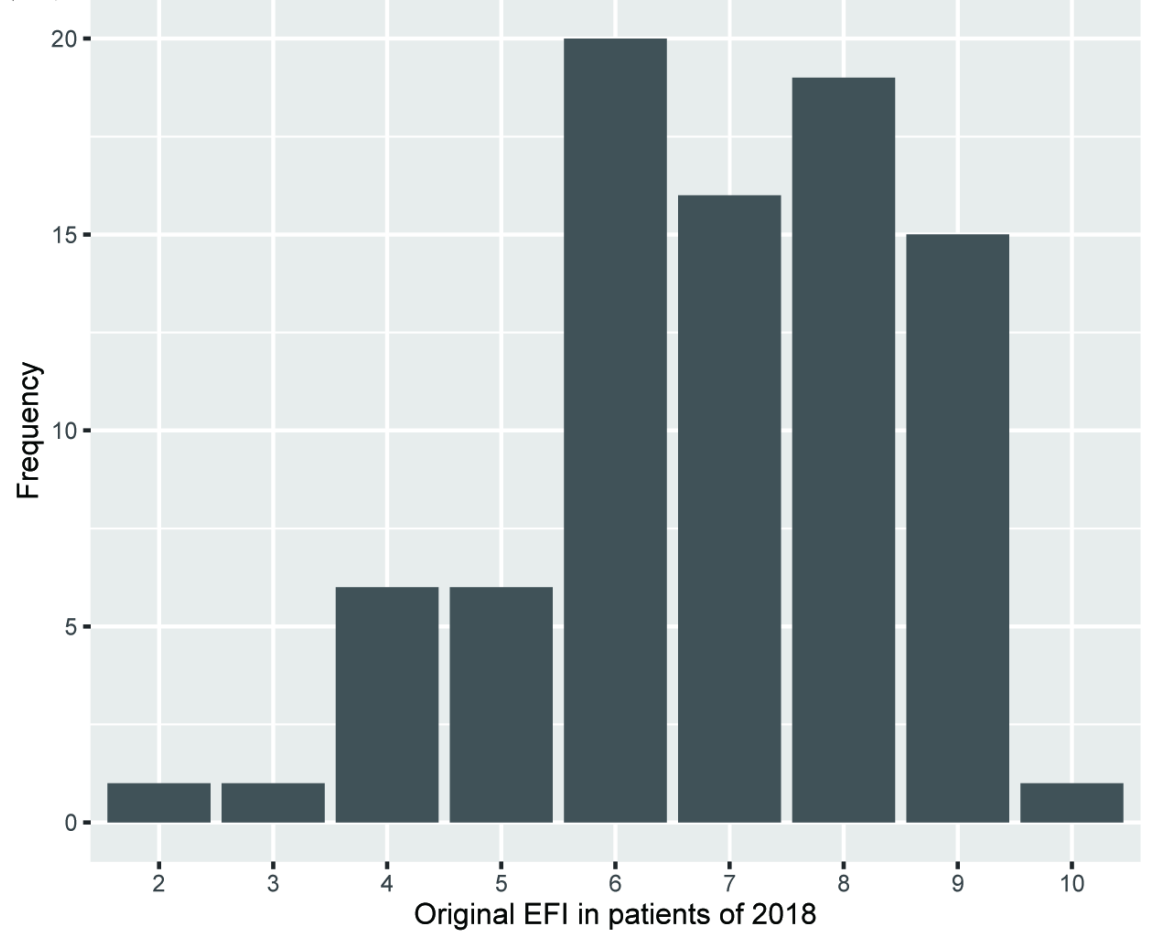

(B)

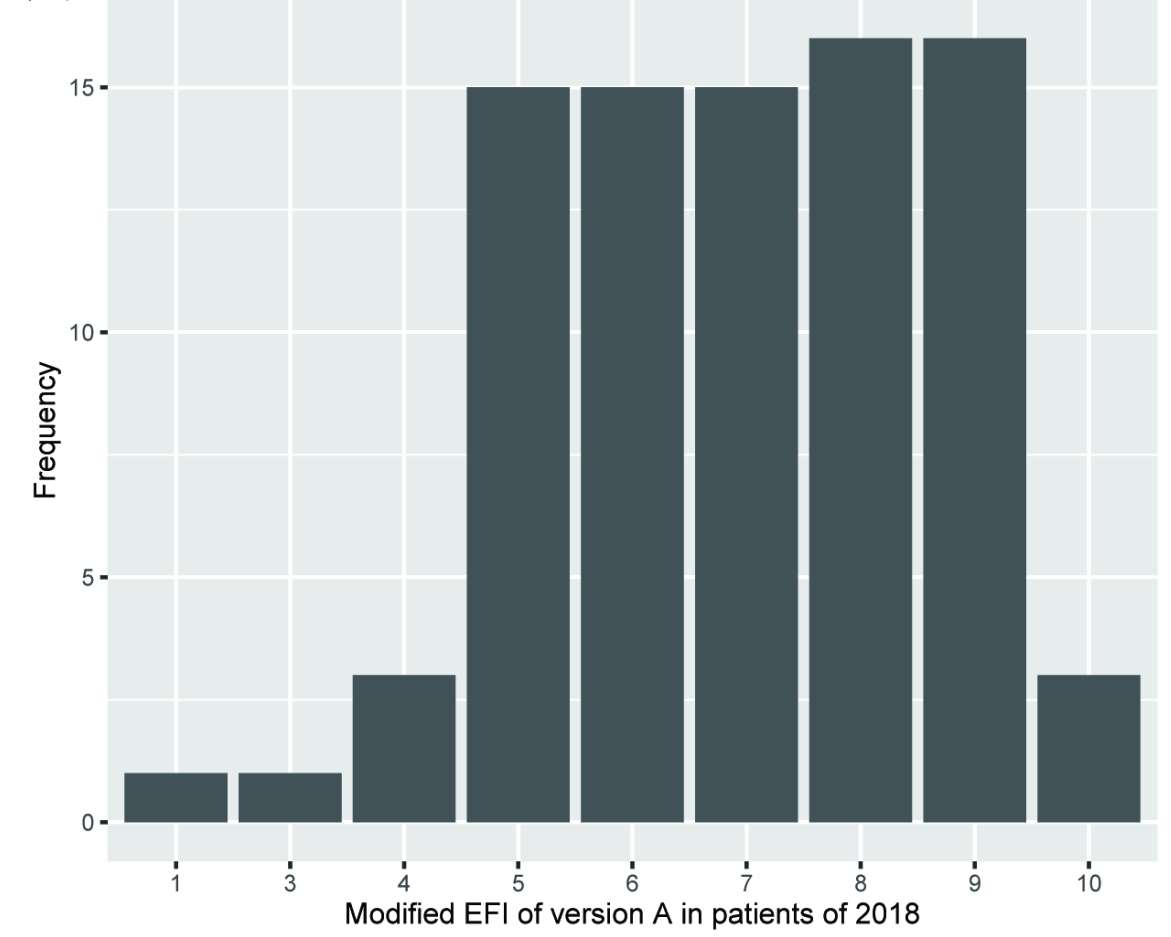

(C)

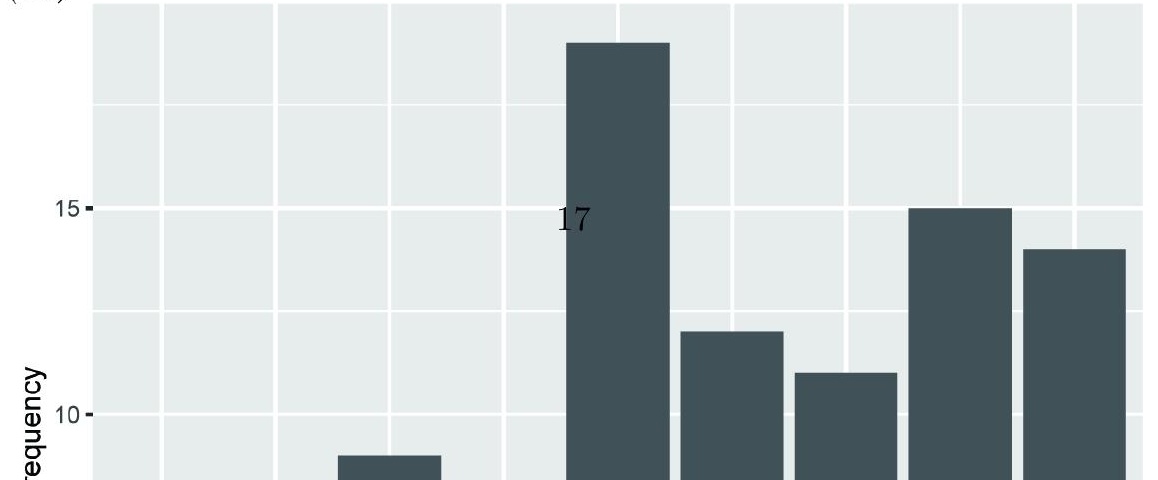

